\title{
OBTENCIÓN DE RATONES ALBINOS EN UN CAMPO MAGNÉTICO PULSANTE DE 5 mT, 60 HZ Y DESARROLLO DE SU MASA CORPORAL
}

\author{
Ivan Ramirez Jiménez ${ }^{1}$ \\ Oscar Barces \\ Sabina Gutiérrez \\ Rocío Coca M.
}

\begin{abstract}
RESUMEN
El trabajo ha consistido en obtener crías de ratones albinos dentro de un campo magnético, de padres sometidos a un campo magnético semicontinuo de $5 \mathrm{mT}$ con pulsos positivos de $60 \mathrm{~Hz}$ orientados en la misma dirección que el campo magnético terrestre, y realizar el seguimiento del desarrollo de dos camadas de ratones. La primera camada de tres ratones durante 65 días y la segunda camada con de 10 ratones, durante un periodo de 55 días.

Se construyo un campo magnético con una bobina de $22 \mathrm{~cm}$ de diámetro, y de ocho $\mathrm{cm}$ de ancho, por la cual se hacia circular una corriente eléctrica de 5 amperios (rms), con la que se obtenía una valor de inducción magnética de 50 gauss.

El desarrollo de la masa corporal de los ratones fueron registrados periódicamente, comparándose los resultados con los datos de ratones del grupo control a tres condiciones de 5,10 y $15^{\circ} \mathrm{C}$ superiores a la temperatura de ambiente y con un mismo programa alimentario, pero con una ligera diferencia de edades por el numero de días de nacidos, que eran de 18 días para el grupo experimental, y 22 días para los ratones del grupo control.

El trabajo experimental ha mostrado que las crías nacidas dentro del campo magnético de 50 gauss tiene una masa ligeramente inferior que las crías nacidas en condiciones naturales.
\end{abstract}

Palabras claves: Campo magnético. Ratones albinos. miliTesla (mT). $\mathrm{Hz}$

\section{SUMMARY}

The work has consisted of obtaining young of mice albinos within a magnetic field, of parents submissive a magnetic field semi - continuing of $5 \mathrm{mT}$ with positive pulses of 60 Hertz oriented in the same direction that the earth's magnetic field, and to make the pursuit of the development of two groups of mice. The first groups of three mice during 65 days and the second group with 10 mice, during a period of 55 days.

The development of the corporal mass of the mice was registered periodically, comparing the results with the data of mice of the group control to three conditions of 5,10 and $15^{\circ} \mathrm{C}$ superior to the temperature of atmosphere and with a same nourishing program, but with a slight difference of ages by the number of days of been born, that were of 18 days for the experimental group, and 22 days for the control group The experimental work has shown that the young born within the magnetic field of 50 Gaussian slightly has an inferior mass that the young born in natural conditions

Key words: Magnetic field, albino mice, miliTesla (mT). $\mathrm{Hz}$

\footnotetext{
${ }^{1}$ Facultad de Ciencias Biológicas, Universidad Ricardo Palma. Av. Benavides 5440. e-mail: iramirez@mail.urp.edu.pe
} 


\section{INTRODUCCIÓN}

Repacholi et al, 1987 han reportado que los campos magnéticos varían en el tiempo generan corrientes eléctricas internas. Por ejemplo, $3 \mathrm{~T} / \mathrm{s}$ puede inducir densidades de corrientes cerca de $30 \mathrm{~mA} / \mathrm{m}^{2}$ alrededor del perímetro de la cabeza humana.

Las densidades de las corrientes eléctricas inducidas pueden usarse como un parámetro firme en la valoración de los efectos biológicos al nivel celular. En lo que se refiere a una valoración del riesgo de salud, es difícil poder correlacionar las densidades de las corrientes en los tejidos, con la intensidad del campo magnético externo.

Sin embargo, es posible calcular, por lo menos dentro de una orden de magnitud, la densidad de flujo magnético que produciría densidades de corrientes potencialmente, peligrosas para los tejidos.

Es factible establecer correlaciones, entre las densidades de los flujos magnéticos producidos por campos sinusoidales homogéneos y los efectos biológicos que producen sobre los cuerpos totalmente expuestos:

a) Entre 1 y $10 \mathrm{~mA} / \mathrm{m}^{2}$ (inducidos por campos magnéticos entre $0.5-5 \mathrm{mT} \mathrm{a}$ $50 / 60 \mathrm{~Hz}$, o entre $10-100 \mathrm{mT}$ a $3 \mathrm{~Hz}$ ), han sido reportados efectos biológicos menores.

b) Entre 10 y $100 \mathrm{~mA} / \mathrm{m}^{2}$ (de 5 - $50 \mathrm{mT}$ a $50 / 60 \mathrm{~Hz}$ o de $100-1000 \mathrm{mT}$ a $3 \mathrm{~Hz}$ ), se han establecido bien los efectos, sobre el sistema visual y nervioso, y ha sido reportado la facilidad de fractura de huesos En este misma información en lo referente a campos magnéticos pulsantes en sus conclusiones manifiestan que: «En el caso de los humanos el umbral de la inducción de los magnetofosfenos, esta entre 2 y 10 $\mathrm{mT}$. y en el rango de frecuencias de 10 $100 \mathrm{~Hz}$. Muchas investigaciones realizados con campos magnéticos de ondas, sinusoidales, cuadradas y pulsantes, han reportado alteraciones, en las células, en los tejidos, y en el sistema animal, cuando la densidad de corriente inducida supera aproximadamente los $10 \mathrm{~mA} / \mathrm{m}^{2}$. Estos efectos reportados incluyen alteraciones en el metabolismo celular en la expresión genética, en las funciones endocrinas y de inmunidad celular, cambio del metabolismo, de las propiedades de crecimiento, y efectos sobre el desarrollo. Sin embargo muchos de estos estudios no han sido reproducidos con éxito.»

A partir de los años 80 se encuentra una enorme variedad de trabajos de investigación sobre la influencia de los campos magnéticos, en los que se experimentan con ratones, haciendo las mas variadas pruebas como por ejemplo; sobre procesos cancerigenos, comportamiento de la sangre, formación de tumores, cicatrización de la piel y muchos otros.

En este contexto, Forgacs' 2003 reportó que el campo variable de $50 \mathrm{~Hz}$ y $100 \mu \mathrm{T}$ ha influido en la producción de cadherina, $y$ de microtubulos, habiendo estos aumentado a lo largo de las superficies de contacto de las células, y un cambio de la forma típica de las células. Madeca 2003 ha reportado que: "la hipótesis de resonancia ciclotrónica propuesta por liboff sobre la oscilación del calcio nunca se ha verificado independientemente a pesar de varios esfuerzos». Es conocido que el calcio es iportante en la formación de microtubulos.

En la literatura citada no hemos encontrado trabajos que confirmen el trabajo de Margineda 2000 que señalaron un efecto claro a campos de 200 microteslas de 50 $\mathrm{Hz}$.

\section{MATERIALES Y MÉTODOS}

Las casas experimentales fueron construidas con tubos de policloruro de vinilo, PVC, y acrílico. Fuente de poder INELPESA DC CONTROLADA 8 A, imput 220 V. AC, output 0-15 V. DC, 0-25 A y para la construcción de la bobina se utilizó alambre de cobre $\mathrm{N}^{\circ} 14$, bobina. Una 
computadora Laptop 220-240 V, Interfase USB para 4 puertos, sensor de campo magnético (60 gauss máx.). Vernier

Se construyó una bobina suficientemente grande para albergar en su interior una jaula de fierro o madera. Esta bobina tiene un diametro interior de $22 \mathrm{~cm}$, un ancho de 8 cm construido con alambre de cobre $\mathrm{N}^{\circ} 14$. (Fotografía 1)

Por la bobina se hizo circular una corriente eléctrica de media onda de 5 amperios, la cual genera inducción de campo magnético de 5.mT. (Fotografía 2). El calor producido por la bobina, aumentó la temperatura de la jaula en $4^{\circ} \mathrm{C}$. sobre la temperatura ambiente. El campo ha sido medido con un sensor magnético de efecto Hall unida a un computador mediante una interfase. El computador dispone de un software Logger Pro de la compañía Vernier.

Se trabajo con 5 grupos de ratones albinos Mus músculus 3 grupos de control, y dos grupos experimentales dentro del campo magnético. En todos los casos primero se obtuvieron las crías a fin de que las condiciones iniciales del control de masa sean homogéneas. Todos deberían tener la misma cantidad de días de nacidos (la misma edad en días).

Los grupos control fueron tres, un grupo de 5 ratones en condiciones ambientales, otro grupo de 3 ratones a $10^{\circ} \mathrm{C}$ mas alto que la temperatura de ambiente, y el tercer grupo de tres ratones a $15{ }^{\circ} \mathrm{C}$ por encima de la temperatura de ambiente.

La toma de datos se hicieron entre los meses de setiembre y diciembre del 2005, en la que la temperatura se mantenía casi constante en $22^{\circ} \mathrm{C}$. Todos los grupos fueron alimentados con el mismo programa que consistía de una dieta de maíz y conejita que se les suministraba dos o tres veces por semana.

Los dos camadas de ratones dentro del campo magnético se trabajaron con un desfase de 43 días.
La primera camada estuvo formado por dos crías, cuyos padres ya habían nacido y vivido dentro de un campo magnético por más de un año. En este grupo se añadió un tercero, nacido de padres en condiciones normales.

El grupo de la segunda camada estuvo constituida de 10 crías, nacidas también de padres provenientes de haberse procreado y vivido dentro de campo magnético de menor intensidad cerca de un año. Estas crías se dividió en dos grupos de 5 ratones. Las crías se obtuvieron en la jaula de madera y para el control del desarrollo corporal a cada grupo se les habilito unos habitáculos de tubos de plástico en forma de U.

A todos los grupos se les hacia una limpieza total se sus casas cada 3 días o semanal. Al mismo tiempo que se hacia la medición de sus masas con una balanza de 0.1 gramo de precisión.

\section{RESULTADOS}

Se han obtenido dos camadas de crías de ratones, una constituida por dos crías, y otra conformada por diez. Estas crías han sido obtenidas de padres que se han desarrollado en un campo magnético prolongado de 60 $\mathrm{Hz}, 5 \pm 4 \% \mathrm{mT}$. Los resultados del desarrollo de la masa corporal se muestran en las tablas del 1- 3

\section{DISCUSIÓN}

Las tablas del control del desarrollo corporal de los ratones, no muestran una tendencia uniforme de ganancia o pérdida de masa corporal. Esto posiblemente se deba al programa de suministro de alimentos que no ha sido estrictamente respetado.

Sin embargo, se ha observado un desarrollo sostenido en el aumento de masa de cada ratón, lo que ha permitido analizar estadísticamente, recurriendo a las regresiones lineales.

Estas regresiones nos han permitido cuantificar el desarrollo corporal de cada 
ratón mediante la velocidades de crecimientos para evaluar el grado de influencia del campo, en el desarrollo corporal.

El grupo de ratones control con temperaturas de 15 grados encima de la temperatura ambiente han sobrevivido dos semanas, pero en el corto tiempo de vida muestran una velocidad de desarrollo de $0.147 \pm 0.96 \%$ g/día y en el drupo control de ratones sometidos a temperatura mayor de $15{ }^{\mathrm{a}} \mathrm{C}$ que la temperatura ambiente también solo vivieron 21 días, llegando a alcanzar una velocidad promedio de $0.304 \pm 22 \%$ g/día.

La colonia de ratones control, a temperatura ambiente, durante las tres primeras semanas llegaron a tener una velocidad de crecimiento $0.10 \mathrm{~g} /$ día (tabla 1 ) Sin embargo es de notar que en esta colonia hay un ratón que alcanza una velocidad de $0.29 \mathrm{~g} /$ día y otro muy bajo de de 0.0036 g/día.

Los ratones control, con temperaturas más altas a la del ambiente, se realizó con la intención de descartar la influencia del aumento de temperatura en la casa experimental debido al calentamiento de la bobina producida por la resistencia ohmica del enrollamiento de la bobina.

La casa se mantuvo aproximadamente a 5 grados por encima de la temperatura de ambiente. La velocidad promedio de crecimiento de la primera camada fue de $0.257 \pm 14 \%$ g/día (tabla 2).

Un valor muy alto, que es necesario seguir analizando con otros experimentos similares para llegar a una conjetura.

La velocidad del desarrollo corporal de la segunda camada en las dos casa experimentales fue de $0.145 \pm 13 \%$ g/día siendo en esta camada, la velocidad de desarrollo más alta, de $0.171 \mathrm{~g} /$ día, y la más baja de 0.08 g/día. (tabla 3)

La velocidad de desarrollo de los ratones dentro del campo magnético desde el punto vista del análisis estadístico, muestra que es ligeramente superior a la velocidad de desarrollo de los ratones control en condiciones de ambiente. Los ratones de la primera camada han crecido 2.7 veces más rápido, y las de la segunda 1.15 veces más rápido.

Sin embargo comparando con los ratones control a las dos diferentes temperatura ya no se puede aseverar lo mismo, mas bien se diría que la rapidez de crecimiento esta influenciado por la temperatura y no por el campo magnético, ya que en ambos casos a las temperaturas de 10 y 15 grados sobre la temperatura de ambiente la tasa de desarrollo de las ratones es mayor con respecto a los de campo que están a una diferencia de temperaturas de 5 y 10 respectivamente.

\section{CONCLUSIONES}

De los resultados se puede concluir que los ratones nacidos en un campo magnético y sometidos en él a los 18 días presentan masas ligeramente menores que las crías nacidas en las condiciones de control. Lo que podría significar una ligera influencia de este campo magnético, sin embargo es necesario realizar mayores estudios.

\section{LITERATURA CITADA}

INTERNATIONAL PROGRAMME ON CHEMICAL SAFETY 1987. Geneva, environmental health criteria 69. Magnetic fields. Published under the joint sponsorship of the United Nations Environment Programme, the International Labour Organisation, and the World Health Organization.

FORGÁCS, Z.. 2003. Effect of $50 \mathrm{~Hz}$ magnetic field exposure on the adherent cell contacts of primary mouse Leydig cells in culture. Volume 47(1-4):27-30. Acta Biologica

NAKASONO, S. 2003 Effects of 50hz, $300 \mathrm{mT}$ sinusoidal magnetic fields on mice behavior and wide-scale gene expression in the whole brain . Tokyo.

MADECA, F. .. 2003. Effects of ELF and static magnetic fields on calcium oscillations in islets of langerhans. University of Bordeaux .France. 
MARGINEDA, J. 2000. Departameno de Fisica.Departamento de Radiologia y Medicina Física. Universidad de Murcia.España,

RAMIREZ, I. et al. 2005.Desarrollo de la masa corporal de ratones albinos Mus musculus sometidos a un campo magnético en un rango de 2 - 25 Gauss. Revista de Ciencias. Volumen II.pp.50-

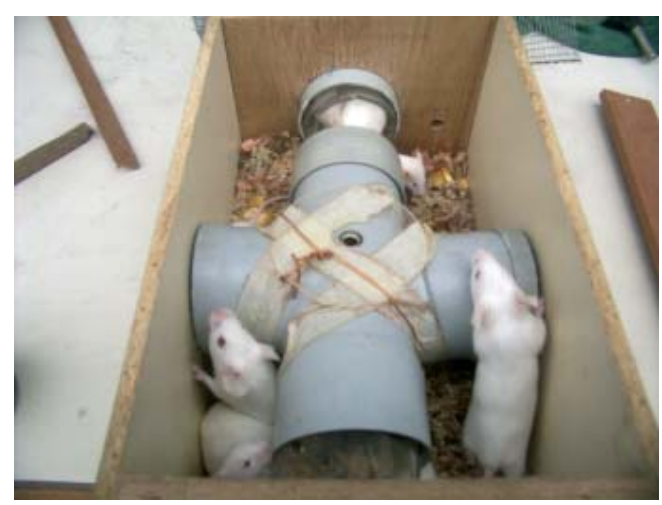

Fotografía 1 Casa campo salón y casa reproducción
69.U.R.P. Dpto. de Académico de Ciencias

LEVIN, M. y ERNST S. G 2000. Applied dc magnetic fields cause alterations in the time of cell divisions and developmental abnormalities in early seaurchin embryos. Dept. of biology. Tufts. university.medford.

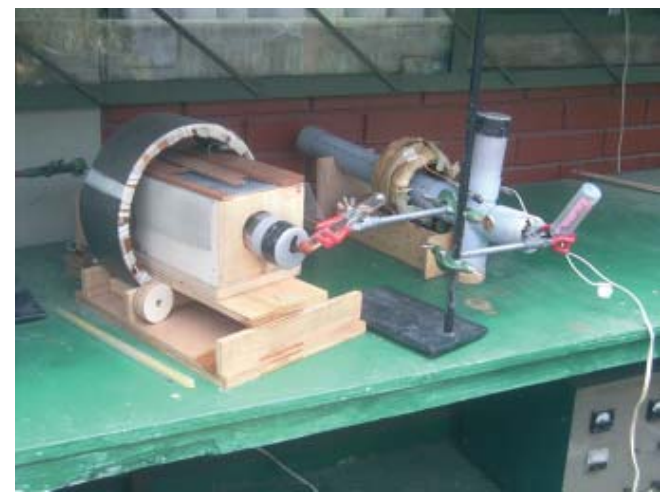

Fotografía 2: Bobina de $22 \mathrm{~cm}$. de radio albergando la casa de madera de reproducción

Tabla 1: Desarrollo de la masa corporal de ratones del grupo control a temperatura ambiente

\begin{tabular}{cc}
\hline $\begin{array}{c}\text { Días de } \\
\text { Nacidos }\end{array}$ & Masa (g) \\
\hline 23 & $8,6 \pm 1,06$ \\
28 & $9,1 \pm 0,60$ \\
30 & $7,8 \pm 0,50$ \\
32 & $8,8 \pm 0,78$ \\
34 & $8,5 \pm 1,08$ \\
35 & $8,5 \pm 1,48$ \\
37 & $8,7 \pm 1,30$ \\
39 & $9,3 \pm 1,31$ \\
41 & $9,7 \pm 1,51$ \\
44 & $9,7 \pm 2,29$ \\
\hline
\end{tabular}


Tabla 2: Masa Corporal de ratones nacidos en campo magnético primera camada

\begin{tabular}{cc}
\hline $\begin{array}{c}\text { Días de } \\
\text { Nacidos }\end{array}$ & Masa (g) \\
\hline $\mathbf{2 5}$ & $9,75 \pm 1.06$ \\
$\mathbf{3 0}$ & $8,45 \pm 0.78$ \\
$\mathbf{3 2}$ & $7,9 \pm 0.85$ \\
$\mathbf{3 4}$ & $7,1 \pm 0.28$ \\
$\mathbf{3 7}$ & $9,55 \pm 1.06$ \\
$\mathbf{3 9}$ & $10,05 \pm 0.35$ \\
$\mathbf{4 1}$ & $9,95 \pm 0.78$ \\
$\mathbf{4 6}$ & $9,75 \pm 0,78$ \\
$\mathbf{4 8}$ & $11,25 \pm 0,49$ \\
$\mathbf{5 1}$ & $11,50 \pm 0.57$ \\
$\mathbf{5 3}$ & $13,50 \pm 0,57$ \\
$\mathbf{5 5}$ & $14,40 \pm 0,14$ \\
$\mathbf{5 8}$ & $14,70 \pm 0,71$ \\
$\mathbf{6 0}$ & $15,80 \pm 1,41$ \\
$\mathbf{6 2}$ & $17,20 \pm 0,85$ \\
$\mathbf{6 5}$ & $16,85 \pm 0,92$ \\
\hline
\end{tabular}

Tabla 3: Ratones procreados, nacidos y desarrollados en campo magnético de 50 G. 60 Hz

\begin{tabular}{ccc}
\hline $\begin{array}{c}\text { Días de } \\
\text { nacidos }\end{array}$ & $\begin{array}{c}\text { Grupo } \\
\text { Experimental } 1 \\
\text { Masa (g) }\end{array}$ & $\begin{array}{c}\text { Grupo } \\
\text { Experimental 2 } \\
\text { Masa (g) }\end{array}$ \\
\hline 0 & $6,02 \pm 0,59$ & $6,20 \pm 0,10$ \\
3 & $5,42 \pm 0,43$ & $5,08 \pm 0,50$ \\
5 & $6,54 \pm 0,69$ & $5.42 \pm 0,08$ \\
7 & $6,10 \pm 0,85$ & $5,38 \pm 0,51$ \\
10 & $6,42 \pm 0,96$ & $6,32 \pm 0,73$ \\
13 & $6,86 \pm 1,04$ & $7,36 \pm 0,74$ \\
17 & $7,06 \pm 1,39$ & $7,06 \pm 1,39$ \\
20 & $7,85 \pm 1,33$ & $8,18 \pm 0,98$ \\
23 & $8,95 \pm 1,32$ & $9,44 \pm 0,99$ \\
27 & $8,43 \pm 1,52$ & $10,22 \pm 1,09$ \\
29 & $11,08 \pm 2,12$ & $10,3 \pm 1,22$ \\
34 & $10,03 \pm 1,53$ & $11,48 \pm 0,50$ \\
41 & $10,28 \pm 2,20$ & $10,10 \pm 1,22$ \\
47 & $11,83 \pm 0,61$ & $9,35 \pm 1,08$ \\
55 & & $11,38 \pm 1,49$ \\
\hline
\end{tabular}

